\title{
Interacción Humano-Computador en la Sociedad Colombiana de Computación
}

\author{
César Alberto Collazos ${ }^{1}$ (D), Wilson J. Sarmiento ${ }^{2}$ iD, Andrés Solano $^{3}$ (D), Yenny A. Méndez ${ }^{4}$ \\ ${ }^{1}$ Universidad del Cauca, Popayán, Colombia \\ ${ }^{2}$ Universidad Militar Nueva Granada, Bogotá, Colombia \\ ${ }^{3}$ Universidad Autónoma de Occidente, Cali, Colombia \\ ${ }^{4}$ Universidad Mayor de Chile, Santiago, Chile \\ ccollazos@unicauca.edu.co,wilson.sarmiento@unimilitar.edu.co,afsolano@uao.edu.co,yenny.mendez@umayor.cl
}

(Publicado en Internet: 1 Diciembre 2020)

La Interacción Humano-Computador (HCI, CHI en USA o IPO en España) en la actualidad está presente en cualquier software o producto y por ende es una asignatura obligatoria en todo el mundo (Abascal et al., 2001). Una interfaz es una "superficie" de contacto (Laurel \& Mountford, 1990), que refleja las propiedades físicas de los usuarios que interactúan, las funciones a realizar, y el balance de poder y control. La interfaz forma parte de un entorno cultural, físico y social, y por tanto, es necesario tener en cuenta una serie de factores al momento de diseñarla. Es así como el diseño de la interfaz se ha convertido en un elemento crítico en el desarrollo de productos software y hardware, y es uno de los principales factores que influyen en el éxito y competitividad de las aplicaciones. Actualmente, los sistemas interactivos prestan una atención cada vez mayor a las interfaces, demandando cambios en el desarrollo de estos productos. Un sistema interactivo ya no es sólo juzgado por su capacidad de realizar operaciones sino también por su capacidad de comunicarlas adecuadamente al usuario.

Por otro lado, las computadoras actualmente tienen características técnicas que les permiten hacer procesamiento y análisis avanzado, y se pueden adquirir a un costo relativamente bajo, lo que permite el desarrollo de productos dirigidos a facilitar la relación entre el hombre y el computador (Doane et al., 1990). El área de Interacción Humano-Computador comienza a ser reconocida como una disciplina dentro del currículo académico en ciencias de la computación a comienzos de los años 80 (Douglas et al., 2002). Desafortunadamente, en la mayoría de los países iberoamericanos esta área no ha evolucionado de la forma como se espera. Muchas razones han influido para que esta área no sea considerada en estos países como parte de formación básica en los currículos actuales tanto en programas tradicionales de Ingeniería Informática, Computación o Sistemas (nombre equivalente a las ciencias de la computación), como en programas emergentes como Ingeniería en Multimedia, en Física, en desarrollo de Videojuegos, entre otros.

Aunque HCI está cada vez más presente en los planes de estudio tanto de pregrado como de posgrado, en la actualidad todavía tiene una presencia poco destacada y, en muchos casos, se detecta la necesidad de incluir o ampliar las materias relacionadas con esta. La reflexión sobre los conocimientos y contenidos relacionados con HCI se hace necesaria para ofrecer una formación y educación estructurada y de calidad, para así formar profesionales que sean capaces de diseñar y construir entornos y productos que se adapten a las necesidades de todas las personas. Los productos, servicios y entornos están diseñados y construidos por personas para ser utilizados por personas, en consecuencia, se hace evidente la necesidad de que los profesionales directamente implicados en el diseño y construcción de estos productos, servicios y entornos, reciban la formación necesaria para generar soluciones eficaces.

Desafortunadamente, al tratar de incluir dentro de alguna estructura curricular el área de HCI, se han presentado algunos inconvenientes por parte de los diversos comités académicos, entre los cuales podemos resaltar 1) la naturaleza básica de HCI, la cual cruza dos disciplinas aparentemente muy diferentes: las ciencias humanas y las ciencias de la información; y 2) Falta de personal altamente capacitado en la formación de esta área. Al respecto es necesario aclarar que aunque se han realizado importantes avances con respecto al último estudio publicado (Collazos, 2005), se requiere mayor número de profesionales. Adicionalmente, no se tiene un acuerdo sobre los tópicos que integran el área y cómo enseñar en los cursos de HCI (Churchill et al., 2013).

Dentro del Simposio Nacional de Maestrías y Doctorados 2020, organizado por la Sociedad Colombiana de Computación en el marco de la celebración de sus 15 años, se observa que el área de HCI comienza a tener un auge muy importante a nivel nacional. Se recibieron diversos trabajos, esencialmente del 
Suroccidente Colombiano, donde se encuentran la mayor cantidad de Grupos de Investigación que trabajan en el área. Las líneas de mayor investigación se concentran en temas relacionados con:

- Usabilidad.

- Metodologías para el Diseño de Sistemas Interactivos.

- Diseño para Personas con Necesidades Especiales (Inclusión).

En el marco del Simposio, entre académicos e investigadores se discutió sobre el futuro cercano del área de HCI al respecto de las temáticas claves, que deberían abordase como parte del currículo y de las investigaciones. Se plantearon las siguientes temáticas generales:

- Trabajo Multidisciplinar.

- Trabajo Colaborativo.

- Inclusión de componentes de ética como parte esencial de la generación de soluciones de TI.

- Relacionar la seguridad de la información con la usabilidad y la accesibilidad.

- Adecuación de herramientas, métodos y procesos de HCI a las características y necesidades que se relacionan con las tecnologías disruptivas.

Adicionalmente, se resaltó la importancia de fortalecer vínculos con el sector empresarial, para que las investigaciones y el nuevo conocimiento adquirido se validen en conjunto con las empresas, y se busque un engranaje que favorezca al crecimiento del área de Interacción Humano-Computador.

Precisamente, buscando sinergias entre el sector académico y el sector empresarial en torno a dos líneas de trabajo, HCI y Diseño de Videojuegos, se desarrolló el conversatorio HCI Games Meeting 2020르. evento organizado por el Capítulo de Interacción Humano-Computador de la Sociedad Colombiana de Computación y el Capítulo Profesional ACM-CHI Cafeteros. El panel en HCI incluyó representantes de la industria nacional del software, empresas colombianas dedicadas a la creación de sistemas interactivos para la industria del entretenimiento, y multinacionales con sus propias divisiones de desarrollo de software, donde el punto común de todas ellas, es que cuentan con un grupo de profesionales dedicados al diseño y desarrollo de interfaces de usuario. Lo primero que se resaltó es el crecimiento, en los últimos cinco años, de los equipos dedicados al área de HCI, tanto en número de personas como en su papel en la cadena de desarrollo de productos. También se señalaron las dificultades del diálogo interdisciplinar y el trabajo articulado con los otros departamentos al interior de las empresas. La principal conclusión del conversatorio fue la creciente demanda de profesionales preparados para el diseño, desarrollo y evaluación de interfaces de usuario y la necesidad de vínculos universidad-empresa, lo cual coincide con reflexiones finales del Simposio Nacional de Maestrías y Doctorados.

Lo anterior, evidencia un punto de inflexión en el país para el área de HCI, dado por su consolidación académica y el crecimiento en la demanda de profesionales, que implica un trabajo en común entre los diferentes actores, que permita sumar esfuerzos y consolidar una comunidad fuerte y cercana a las necesidades de país y la región, con una visión globalizada de cara a los nuevos retos de la quinta revolución industrial.

\section{Referencias}

Abascal, J., Aedo, I., Cañas, J., Gea, M., Gil, A. B., Lorés, J., Martínez, A. B., Ortega, M., Valero, P., \& Vélez, M. (2001). La interacción persona-ordenador (J. Lorés (ed.); Primera Ed). AIPO, Asociación Interacción Persona Ordenador. https://aipo.es/libro/pdf/00Portad.pdf

Churchill, E. F., Bowser, A., \& Preece, J. (2013). Teaching and learning human-computer interaction. Interactions, 20(2), 44-53. https://doi.org/10.1145/2427076.2427086

Collazos, C. (2005). La enseñanza de HCI en Colombia. I Jornadas de Trabajo Sobre Enseñanza de HCI, 81-91.

Doane, S. M., Pellegrino, J. W., \& Klatzky, R. L. (1990). Expertise in a Computer Operating System: Conceptualization and Performance. Human-Computer Interaction, 5(2-3), 267-304. https://doi.org/10.1080/07370024.1990.9667156

Douglas, S., Tremaine, M., Leventhal, L., Wills, C. E., \& Manaris, B. (2002). Incorporating Human-Computer Interaction into the undergraduate computer science curriculum. ACM SIGCSE Bulletin, 34(1), 211-212.

\footnotetext{
${ }^{1}$ Enlace al video de la transmisión del HCI Games Meeting 2020: https://youtu.be/4QM8fs_83j8
} 
https://doi.org/10.1145/563517.563419

Laurel, B., \& Mountford, S. J. (1990). The Art of Human-Computer Interface Design. Addison-Wesley Longman Publishing Co. https://dl.acm.org/doi/book/10.5555/575201 